\title{
Carbon Nanotube Ring Oscillator for Detecting lonized Radiation
}

\author{
Yaser Mohammadi Banadaki ${ }^{1 *}$, Safura Sharifi ${ }^{2,3}$, Walter O. Craig III $^{1}$ and Hsuan-Chao $\mathrm{Hou}^{3}$ \\ 1. College of Science and Engineering, Southern University and A\&M College, Baton Rouge, LA 70813, USA \\ 2. College of Science, Louisiana State University, Baton Rouge, LA 70803, USA \\ 3. Department of Electrical and Computer Engineering, Louisiana State University, Baton Rouge, LA 70803, USA
}

\begin{abstract}
In this paper, we have explored the feasibility of a carbon nanotube (CNT) ring oscillator (RO) for detecting ionized radiation. The effect of ion irradiation on the oscillation frequency of CNT-based RO is considered using the displacement damage dose (DDD) methodology. The analytical model of the irradiated resistance of metallic single-walled CNT (SWCNT) has been developed and verified by experimental data for increasing DDD from $10^{12}$ to $10^{17} \mathrm{MeV} / \mathrm{g}$. We have found that 100 times increase in the DDD from $10^{15}$ to $10^{17} \mathrm{MeV} / \mathrm{g}$ results in nearly 20 times increase in propagation delay of an input signal passing through $500 \mathrm{~nm}$ metallic SWCNT, which can be easily read. It is also found that an order of magnitude increase in the length of SWCNT results in approximately an order of magnitude decrease in the minimum range of detectable DDD considering the oscillation frequency of CNT RO as the output of the proposed radiation detector. As carbon nanotube with the record length of $50 \mathrm{~cm}$ has been reported, it is very promising for detecting much lower radiation.
\end{abstract}

Key words: Carbon nanotube, ring oscillator, radiation detection, displacement damage dose.

\section{Introduction}

The energy per decay of nuclear radiation can be very small, but the energy released in a nuclear process is larger than the atomic binding energies and thereby any emitted particles will have sufficient energy to directly ionize or excite atoms, known as ionizing radiation [1]. The detector with highest resolution and low ionization potential is required to detect the primary ionization of incident radiation [2]. Ionization-based detectors have mostly used gases as the active medium, but the drawbacks of gases are low density of the stopping material and large effective ionization potential $(\sim 20 \mathrm{eV})$. For solid state detector, the primary ionization must be collected to make a direct measurement of the energy of nuclear radiation. Thus, metals and insulator are not applicable as the generated electron and positive ion pairs are rapidly recombined in metal and insulators, making difficult

"Corresponding author: Yaser Mohammadi Banadaki, Ph.D., assistant professor, research field: emerging technologies for high-performance circuits and sensors. the collection of the charged particles. Semiconductor-based ionization sensors are usually 1,000 times denser and its ionization potential is 10 times smaller than gases-based ionization sensors. However, silicon-surface barrier detectors has large area and thereby a large capacitance, resulting in its low resolution. Germanium diode detectors have the highest resolution of any direct ionization devices due to its small bandgap and small effective ionization potential. However, its small band gap also makes the electro/hole pairs collection sensitive to thermal fluctuation noise, which exponentially relates to the size of band gap.

Carbon has been widely tipped as a promising element for next-generation electronics due to its impressive allotropes [3-6]. Among carbon allotropes, carbon nanotube (CNT) [7, 8] and graphene [9-11] are prominent contenders for high-performance sensors. CNT has limited number of atoms in its circumference, thus removal of one atom can significantly change the carrier transport. When 
radiations interact with $\mathrm{CNT}$, the total number of created ion-pairs is very small on a macroscopic scale, and thereby ionization of charged particles is less important than the increase of scattering phenomena due to the generated defects in the lattice [12]. This together with low ionization potential and compact size of CNT-based ionization detectors results in its superior energy resolution. This makes it to be a very promising material for detecting the primary ionization of incident radiation [8]. The mobility of carbon nanotube is much higher than germanium and silicon, which allows effective compensation of the defect and impurities scattering in carrier transport. The chemical structure of the CNT lattice can be significantly and permanently changed by a single energetic ion. Similar to carbon, all type of solid-state detectors can be damaged by long exposure to the charged particles because the lattice will be disrupted and poisoned by the presence of many defects in the lattice. The CNT-based radiation sensor can be designed similar to polymeric material and mineral mica, which are particularly sensitive to radiation damage and very efficient detectors of rare events that produce highly ionizing radiation. In these materials, the produced crack is localized on the molecular scale and can be expanded by chemical etching to the microscopic scale. However, the CNT-based detectors are conductor and can be fabricated for on-chip nuclear radiation detection. In addition, the electronic pulses in response to the ionizing radiation in most detectors need to be processed by nuclear instrument modules to measure the time and height of the produced signal. This radioactive incident rapidly decays away after being produced and thereby the collection of data must be as rapidly as possible usually during a very limited time. On the other hand, the produced defects in CNT-based radiation sensor are permanent and can be detected without loss of signal in an event of primary radiation.

The output of most detectors is the height or length of an output signal that carries information about the energy departed in the detector. The output signal must be preliminary amplified before being sent through a coaxial cable to a linear amplifier to prevent the noise in the cable from destroying the tiny detector signal. However, if the metallic SWCNTs are used as interconnects in a ring oscillator (RO) circuit, the change in the resistance of CNT interconnects by the radiation exposure can be converted to the change in oscillation frequency as an observant of radiation dose. The oscillation has been taken spontaneously in the close loop of ring oscillator and there is no need to produce a reference input signal to detect radiation dose. This reduces the complexity of detection circuits. The data analyzed system can be a simple tunable RC filter on the chip which measures the oscillation frequency without any degradation of signal height. The importance of CNT-based ring oscillator is not only their mechanism of action, but also the speed and accuracy of ionized radiation detection in a much smaller package than current-generation radiation sensors.

The rest of the paper is organized as follows. Section 2 presents an analytical model of the irradiated resistance of metallic SWCNT. Section 3 discusses the verification of the developed model for exposure to different ion irradiations considering the displacement damage dose (DDD) methodology. Then, the feasibility of a metallic SWCNT as a radiation detector has been demonstrated. The last section draws summarizing conclusions.

\section{Model Equations}

It is predicted that metallic CNTs have less problem with electromigartion than copper interconnects. The CNTs exhibit high thermal and electrical conductivity $[13,14]$. Fig. 1a shows the equivalent circuit model of a metallic SWCNT isolated above a ground plane [15], which consists of circuit elements distributed along its length. In the model, the inductance $(L)$ consists of the series of magnetic inductance $\left(L_{M}\right)$ and kinetic inductance $\left(L_{K}\right)$ as follows: 
(a)
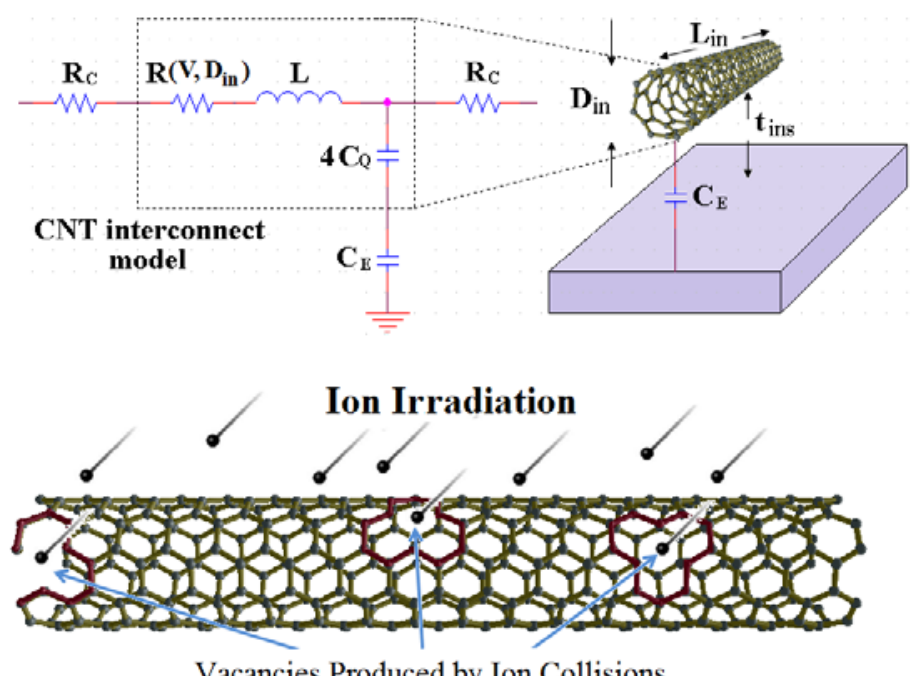

(b)

Vacancies Produced by Ion Collisions

Fig. 1 (a) Transmission line model of a SWCNT interconnect and (b) schematic of metallic CNT imparted by ionization radiation.

$$
\begin{gathered}
L_{M}=\frac{10^{-6}}{2 \pi} \ln \left(\frac{t_{\text {ins }}}{D_{i n}}\right) \\
L_{k} \cong \frac{\pi \hbar}{e^{2} v_{F}}
\end{gathered}
$$

where, $t_{i n s}$ is the distance between nanotube and the ground, $D_{i n}$ is SWCNT diameter, $v_{F}$ is Fermi velocity, $\hbar$ is reduced Planck constant and $e$ is electron charge. The capacitance also consists of quantum capacitance of the nanotube and electrostatic capacitance between a wire and a ground plane as follows:

$$
\begin{gathered}
C_{Q} \cong \frac{e^{2}}{\pi \hbar v_{F}} \cong 100 a F / \mu m \\
C_{E} \cong \frac{2 \pi \varepsilon}{\ln \left(\frac{t_{i n s}}{D_{i n}}\right)}
\end{gathered}
$$

where, $\varepsilon$ is dielectric constant of substrate. The resistance, $R$ in the equivalent transmission model can be determined by the effective mean free path of electrons, $\lambda_{\text {eff }}$ as follows:

$$
R=\frac{R_{Q}}{4} \frac{L_{\text {in }}}{\lambda_{\text {eff }}}
$$

where, $R_{Q}=\pi \hbar / 2 e^{2} \cong 12.9 k \Omega$ is quantum resistance.

The effective mean free path includes the effects of scattering mechanisms due to acoustic phonons, optical phonons and the defects (vacancies) produced by ion irradiation. The spontaneous scattering lengths for emitting an acoustic phonon $\left(\lambda_{a c}\right)$ can be estimated as follows [16]:

$$
\lambda_{a c}=400.46 \times 10^{3} \frac{D_{i n}}{T}
$$

where, $T$ is temperature. The mean free path (MFP) due to optical phonons, $\lambda_{o p}$ includes three scattering lengths as follows:

$$
\frac{1}{\lambda_{o p}}=\frac{1}{\lambda_{o p, e m s}^{a b s}}+\frac{1}{\lambda_{o p, e m s}^{\text {field }}}+\frac{1}{\lambda_{o p, a b s}}
$$

where, the three scattering lengths are optical phonon absorption $\left(\lambda_{o p, a b s}\right)$, optical phonon emission for the absorbed energy ( $\lambda_{o p, e m s}^{a b s}$ ), and optical phonon emission for the electric field across the SWCNT length $\left(\lambda_{\text {op }, \text { ems }}^{\text {fien }}\right)$, which can be calculated as follows [14]:

$$
\begin{gathered}
\lambda_{o p, a b s}=\frac{N_{o p}(300)+1}{N_{o p}(T)} \lambda_{o p}^{0} \\
\lambda_{o p, e m s}^{a b s}=\lambda_{o p, a b s}+\frac{N_{o p}(300)+1}{N_{o p}(T)+1} \lambda_{o p}^{0} \\
\lambda_{o p, e m s}^{\text {field }}=\frac{\hbar \omega_{o p}-k_{B} T}{q \frac{V}{L_{i n}}}+\frac{N_{o p}(300)+1}{N_{o p}(T)+1} \lambda_{o p}^{0}
\end{gathered}
$$

where, $\lambda_{o p}^{0}=56.4 D_{i n}$ is the spontaneous scattering length for emitting an optical phonon, $N_{o p}=1 /\left[1+\exp \left(\hbar \omega_{o p} / k_{B} T\right)\right]$ is the optical phonon occupation in Bose-Einstein statistics, $k_{B}$ is the 
Boltzmann constant, $\hbar \omega_{o p}$ is optical phonon energy and $L_{i n}$ is the CNT interconnect length.

The effects of SWCNTs exposure to different ion irradiation (protons, alpha-particles, and carbon ions) can be incorporated in the model with the DDD methodology [12], which accounts for the total energy imparted by radiation to the circumference of SWCNT interconnects. The vacancies are randomly formed throughout the SWCNT lattice as conceptually shown in Fig. 1b. Unlike the atoms of materials with 3D bulky lattice, SWCNTs are 3D thin-film volume and thereby every atom is effectively exposed to ion irradiation and can potentially contribute in vacancy formation. The number of vacancies per unit mass of SWCNT is proportional to the energy imparted to the lattice and can be calculated by dividing DDD by the energy required to produce a vacancy as follows [12]:

$$
V_{L}=\frac{D D D m_{L}}{E_{\text {vac }}}
$$

where, $E_{v a c}=\left(E_{b}+E_{t h} / 0.4\right)$ is the energy required to produce a vacancy, the threshold energy, $E_{t h}$ is the minimum energy required to create a vacancy in a SWCNT, $\quad E_{b}=3 \mathrm{eV}$ is carbon-carbon binding energy. The SWCNT mass per unit length can be calculated by $m_{L}=W_{c} N_{c} / l_{c}$, where $W_{c}$ is the atomic weight of carbon, $N_{c}$ is the number of atoms per circumferential unit cell around a SWCNT and $l_{c}$ is the length of the circumferential unit cell. Assuming the contribution of all produced vacancies on carriers transport, the inverse of $V_{L}$ is the average length of vacancy-free segments along the SWCNT and can be define as the MFP of ion irradiation, $\lambda_{\text {Rad }}=V_{L}^{-1}$.

The effective mean free path can be calculated by Matthiessen's rule considering all the scattering mechanisms as follows:

$$
\frac{1}{\lambda_{\text {eff }}}=\frac{1}{\lambda_{a c}}+\frac{1}{\lambda_{o p}}+\frac{1}{\lambda_{\text {Rad }}}
$$

The effective MFP, $\lambda_{\text {eff }}$ determines the resistance, of the SWCNT due to both the pre-irradiation terms of acoustic and optical phonons scattering mechanisms and the contribution due to defects produced by ion irradiation.

\section{Results and Discussion}

\subsection{Model Verification}

Fig. 2a shows the change in the ratio of the irradiated resistance, $\mathrm{R}(\mathrm{DDD})$ to the pre-irradiation resistance, $\mathrm{R}(0)$ as a function of increasing the displacement damage dose. In order to test the accuracy of our analytical model, the fitted curve of several experimental results in [12] has been also shown in the figure. It can be seen that our model is in correlation with the experimental results for the same SWCNT $(10,10)$ with diameter of $1.35 \mathrm{~nm}$, showing the same increase in resistance with increasing DDD. Fig. $2 \mathrm{~b}$ shows the total resistance versus voltage bias across $10 \mu \mathrm{m}$ length $\operatorname{SWCNT}(10,10)$ as a function of increasing DDD from $10^{12} \mathrm{MeV} / \mathrm{g}$ to $10^{17} \mathrm{MeV} / \mathrm{g}$. It can be seen that increasing the resistance is significant for higher values of DDD. It becomes constant with bias voltage as the mean free path of ion irradiation shows the dominant scattering mechanism (voltage independent term in Eq. (12)).

\subsection{CNT-Based Inverter-Pair Radiation Detector}

Fig. 3a shows the schematic of a CNT-based inverter pair, which can be implemented by CNT FETs and a CNT Interconnect. The CNT interconnect has been simulated using the developed model in Section 2 together with the CNT FET with the developed semi-classical model in [17]. Fig. 3b shows that the propagation delay of CNT inverter pair can be sensitive output for measuring the DDD imparted to the CNT interconnect if its length has been chosen depending on the range of DDD variation. It can be seen that a longer CNT interconnect can detect a lower DDD. For example, an order of magnitude increase in DDD from $10^{14}$ to $10^{15}$ results in approximately an order of magnitude variation in propagation delay from $\mathrm{t}_{\mathrm{pd}}=1.4 \times 10^{-8}(\mathrm{~s})$ to $10^{-7}(\mathrm{~s})$ for $50 \mu \mathrm{m}$ length CNT interconnect (green). However, 

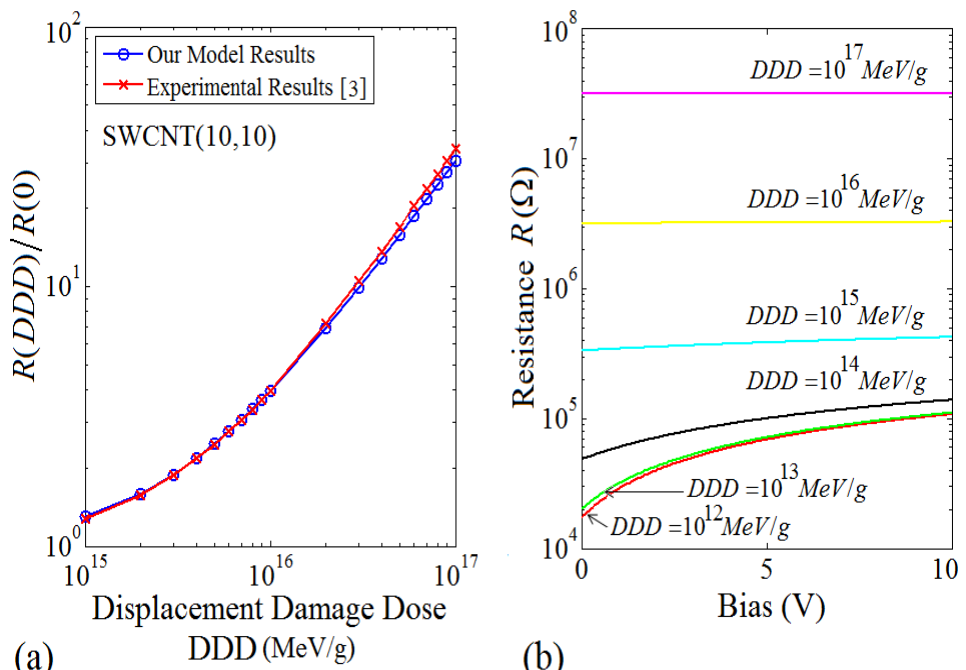

(b)

Fig. 2 (a) Change in the ratio of the irradiated resistance, R(DDD) to the pre-irradiation resistance, R(0) as a function of increasing the displacement damage dose. The curve of experimental results in [12] is shown for comparison. (b) Total resistance versus voltage bias across $10 \mu \mathrm{m}$ length $\operatorname{SWCNT}(10,10)$ as a function of increasing DDD from $10^{12}$ to $10^{17} \mathrm{MeV} / \mathrm{g}$. The increase in resistance is significant for higher values of DDD and it becomes constant with bias voltage as the MFP of ion irradiation becomes dominant scattering mechanism.

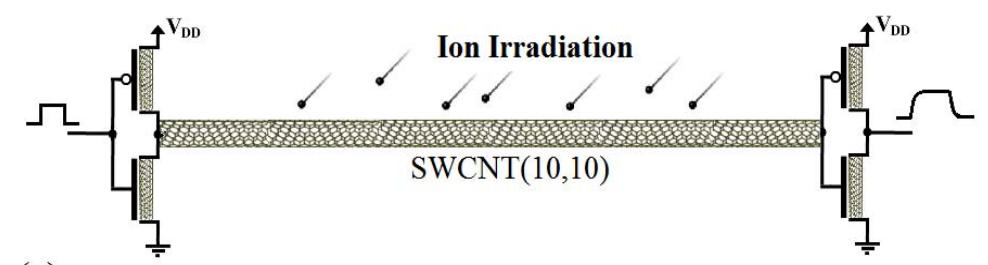

(a)

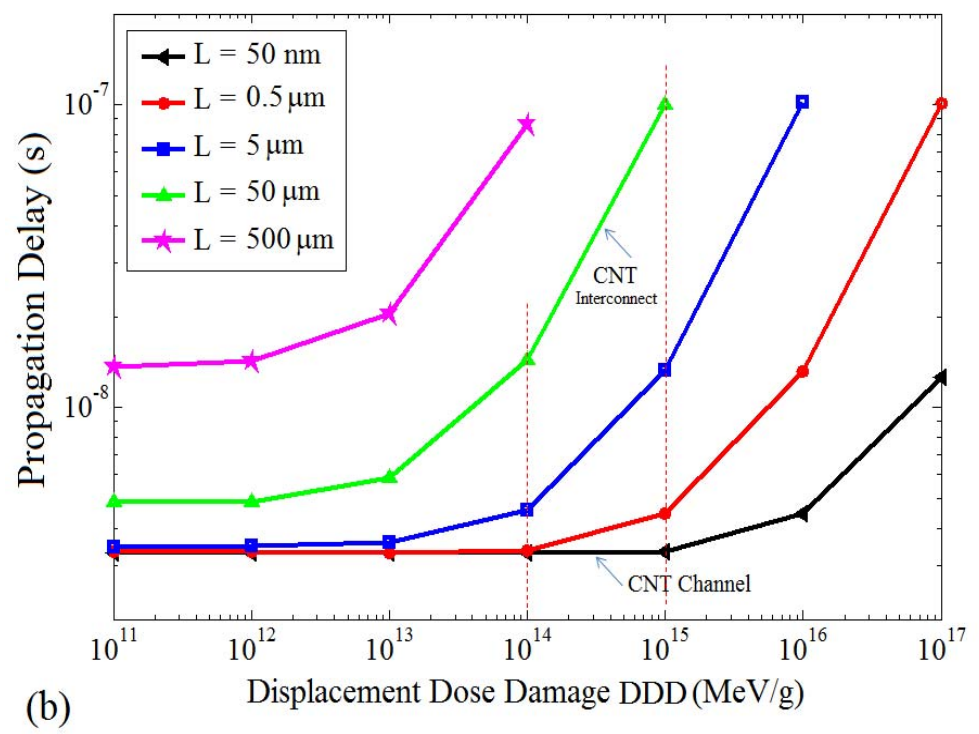

Fig. 3 (a) CNT-based inverter pair including a metallic SWCNT(10,10) interconnect in between two CNT FET inverters. (b) Propagation delay of CNT inverter pair versus displacement damage dose for different CNT lengths. Note: Semiconducting CNT with $50 \mathrm{~nm}$-length and CNT interconnect with $50 \mu \mathrm{m}$-length have been shown in the range of DDD variation from $10^{14}$ to $10^{15} \mathrm{MeV} / \mathrm{g}$. The contact resistance between metal and CNT has been considered equal to $24 \mathrm{k} \Omega$. 
CNT with the length of $50 \mathrm{~nm}$ (black) has no variation in its propagation delay for the same variation in DDD. As such, for CNT channel with much smaller length than CNT interconnect, the effects of DDD on the operation of CNT FET is negligible while ionized radiation results in significant variation of the propagation delay associated with the resistance variation of CNT interconnect. In this work, the length of CNT channel is considered $16 \mathrm{~nm}$ and thereby the effect of DDD on the output variable, i.e. propagation delay, has been neglected.

\subsection{CNT-Based Ring-Oscillator Radiation Detector}

In a ring oscillator, the input of each inverter is the output of the previous inverter. The ring closes by returning the output of the last inverter to the first stage. Fig. 4a shows the cascade combination of three CNT-based inverters and CNT interconnects, which results in sequentially change of logic state and consequently the signal oscillation. The frequency of oscillation depends on the propagation delay $\left(t_{p}\right)$ in voltage switching between successive inverter stages and can be determined by $f=1 /\left(2 N t_{p}\right)$, where $N$ is the number of stages. The resistance of SWCNT interconnects are increased by increasing radiation, which results in increase in $\mathrm{RC}$ delay and consequently the reduction in the oscillation frequency. As it has been explained in Section 3.2, the length of CNT channel is much smaller than CNT interconnect and thus the effect of DDD on the oscillation frequency can be neglected.
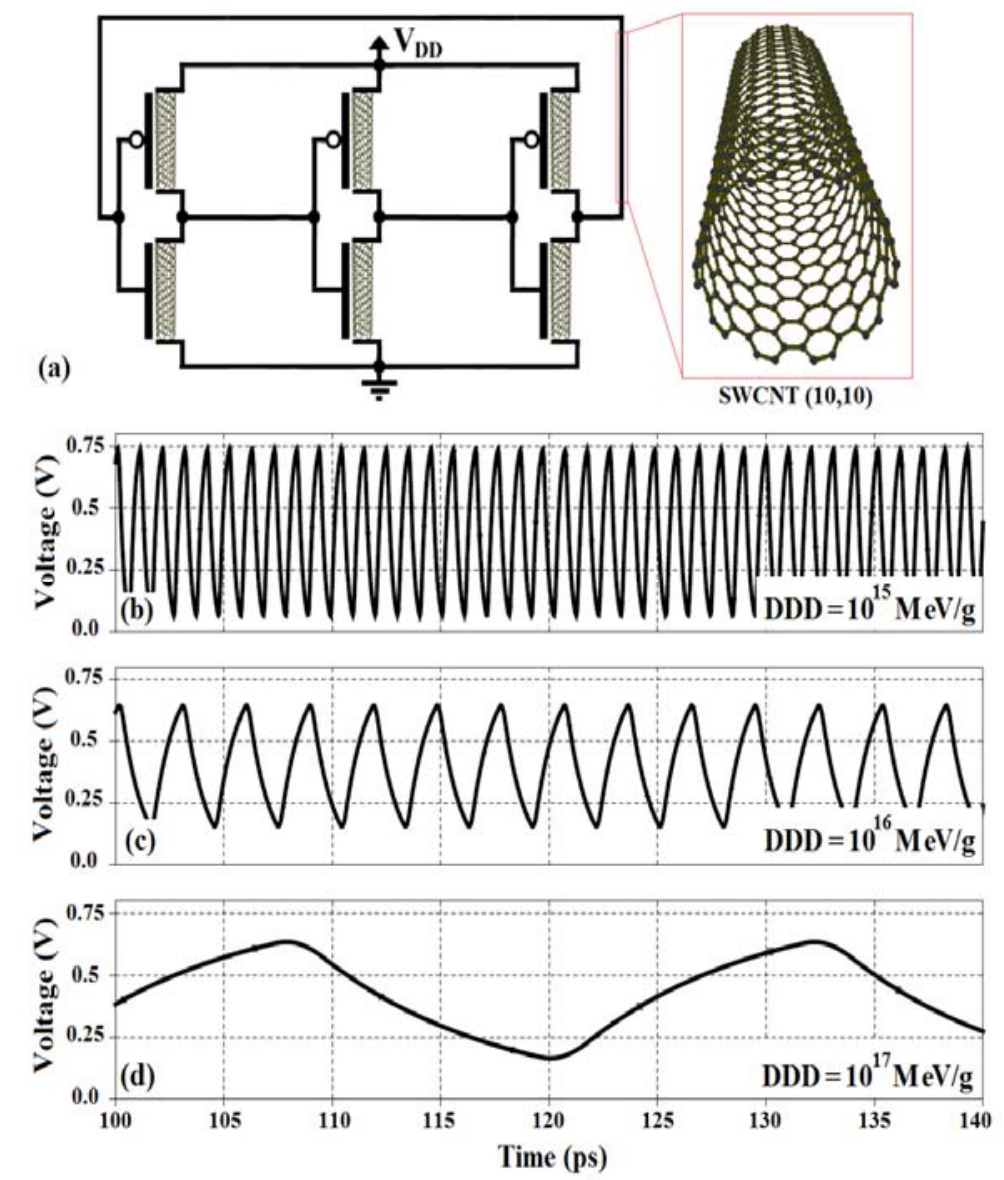

Fig. 4 (a) CNT-based ring oscillator consisting six CNTFET and three CNT interconnects. (b) Output waveforms of the CNT-based ring oscillator for exposure to ion radiation with three displacement density dose of (b) $10^{15}$, (c) $10^{16}$ and (d) $10^{17} \mathrm{MeV} / \mathrm{g}$. 


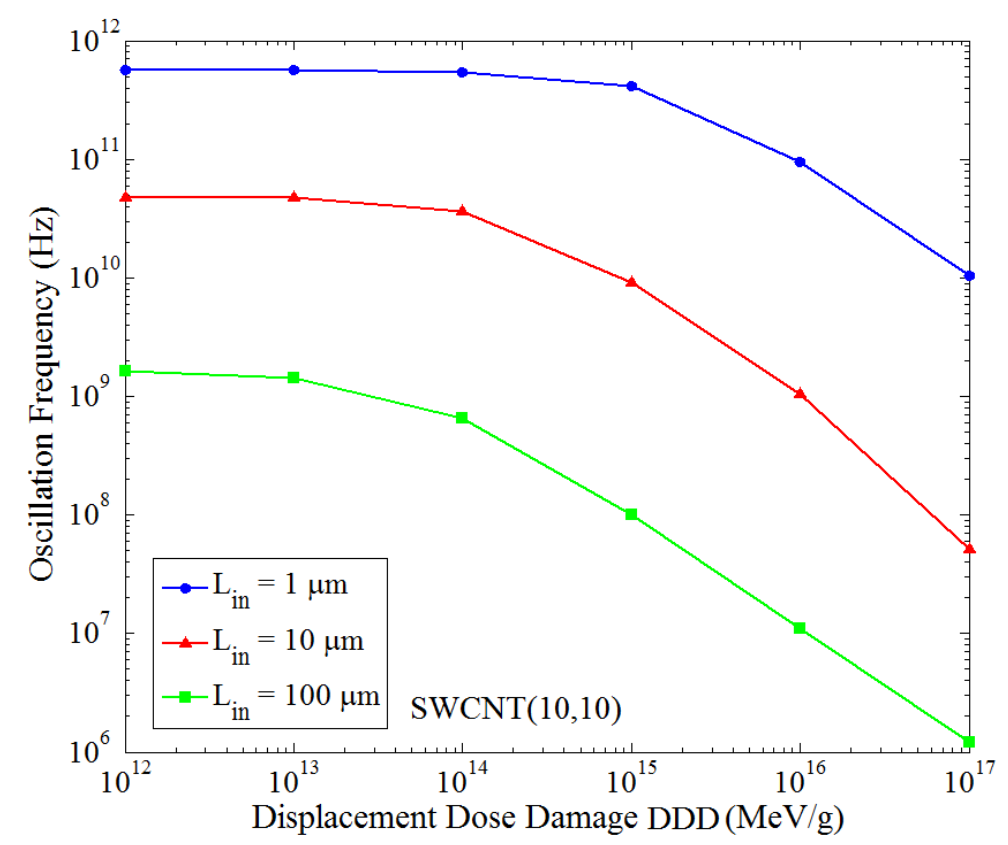

Fig. 5 Oscillation frequency of CNT-based RO versus DDD for metallic SWCNT $(10,10)$ interconnect with three different lengths of 1,10 and $100 \mu \mathrm{m}$.

The pre-radiation oscillation frequency of CNT-based RO is obtained $\mathrm{f}=1.1 \mathrm{THz}$ considering $0.5 \mu \mathrm{m}$ length SWCNT $(10,10)$. The output waveforms of the circuit for exposure to ion radiation with three displacement density dose of $10^{15}, 10^{16}$ and $10^{17} \mathrm{MeV} / \mathrm{g}$ are shown in Fig. 4b, 4c and 4d, respectively. It can be seen that two orders of magnitude increase in the DDD results in roughly three orders of magnitude decrease in oscillation frequency.

Fig. 5 shows the oscillation frequency versus DDD for SWCNT $(10,10)$ interconnect with three different lengths of $1 \mu \mathrm{m}, 10 \mu \mathrm{m}$, and $100 \mu \mathrm{m}$. It can be seen that the CNT-based RO with $1 \mu \mathrm{m}$-length SWCNT can be only used for the detection of the DDD above $10^{15} \mathrm{MeV} / \mathrm{g}$ while longer SWCNTs can detect lower DDD. For instance, the detection of DDD $=10^{13}$ $\mathrm{MeV} / \mathrm{g}$ with 100 times longer SWCNT $(100 \mu \mathrm{m})$ has the same accuracy as the detection of DDD $=10^{15}$ $\mathrm{MeV} / \mathrm{g}$ with $1 \mu \mathrm{m}$-length SWCNT interconnects. As carbon nanotube with the record length of $50 \mathrm{~cm}$ has been reported [18], CNT is very promising for application in the detection of much lower DDD.

\section{Conclusions}

The feasibility of a metallic single-walled carbon nanotube as an ionized radiation detector has been investigated. An analytical model of the irradiated resistance of SWCNT interconnect has been presented using DDD methodology and verified against experimental data for exposure to different ion irradiations. By designing CNT-based ring oscillator, the variation in oscillation frequency due to the increase in scattering of carriers in CNT interconnect has been used as an observant of radiation dose. It has been found that the propagation delay of CNT interconnect is very sensitive to DDD, such that two orders of magnitude increase in the DDD results in roughly three orders of magnitude decrease in oscillation frequency. In addition, CNT with different length can be used for detecting different DDD ranges, such that a longer CNT interconnect needs to be used for lower DDD detection. Carbon nanotube is promising material for detection of very small DDD as very long CNT has been recently produced. 


\section{References}

[1] Knoll, G. F. 2010. Radiation Detection and Measurement. John Wiley \& Sons.

[2] Tsoulfanidis, N. and Landsberger, S. 2015. Measurement and Detection of Radiation. CRC Press.

[3] Banadaki, Y. and Srivastava, A. 2015. "Scaling Effects on Static Metrics and Switching Attributes of Graphene Nanoribbon FET for Emerging Technology." IEEE Transactions on Emerging Topics in Computing 3 (4): 458-69.

[4] Srivastava, A., Marulanda, J. M. and Xu, Y. et al. 2015. Carbon-based Electronics: Transistors and Interconnects at the Nanoscale. Pan Stanford.

[5] Banadaki, Y. M. and Srivastava, A. 2015. "Investigation of the Width-Dependent Static Characteristics of Graphene Nanoribbon Field Effect Transistors Using Non-Parabolic Quantum-Based Model.” Solid-State Electronics 111: 80-90.

[6] Banadaki, Y. M. and Srivastava, A. 2013. "A Novel Graphene Nanoribbon Field Effect Transistor for Integrated Circuit Design." In IEEE 56th International Midwest Symposium on Circuits and Systems (MWSCAS), 924-7.

[7] Mohsin, K., Banadaki, Y. and Srivastava, A. 2014. "Metallic Single-Walled, Carbon Nanotube Temperature Sensor with Self Heating." In SPIE Smart Structures and Materials 906003-906003-7.

[8] Ma, J., Yeow, J. T. and Chow, J. C. et al. 2007. "A Carbon Nanotube-Based Radiation Sensor.” International Journal of Robotics \& Automation 22 (1): 49.

[9] Banadaki, Y., Mohsin, K. and Srivastava, A. 2014. "A Graphene Field Effect Transistor for High Temperature Sensing Applications.” In SPIE Smart Structures and Materials 90600F-90600F-7.
[10] Koybasi, O., Childres, I. and Jovanovic, I. et al. 2012. "Graphene Field Effect Transistor as a Radiation and Photodetector." In SPIE Defense, Security and Sensing 83730H-83730H-8.

[11] Banadaki, Y. M., Srivastava, A. and Sharifi, S. 2016. "Graphene Nanoribbon Field Effect Transistor as Nanometer-Size on-Chip Temperature Sensor." In SPIE Smart Structures and Materials.

[12] Rossi, J. E., Cress, C. D. and Helenic, A. R. et al. 2012. "Ion Irradiation of Electronic-Type-Separated Single Wall Carbon Nanotubes: A Model for Radiation Effects in Nanostructured Carbon." Journal of Applied Physics 112 (3): 034314.

[13] Chen, F., Joshi, A. and Stojanović, V. et al. 2007. "Scaling and Evaluation of Carbon Nanotube Interconnects for VLSI Applications." In Proceeding of the 2nd international conference on Nano-Networks.

[14] Mohsin, K. M., Srivastava, A. and Sharma, A. K. et al. 2013. "A Thermal Model for Carbon Nanotube Interconnects." Nanomaterials 3 (2): 229-41.

[15] Srivastava, A., Xu, Y. and Sharma, A. K. 2010. "Carbon Nanotubes for Next Generation Very Large Scale Integration Interconnects." Journal of Nanophotonics 4 (1): 041690-041690-26.

[16] Franklin, A. D. and Chen, Z. 2010. "Length Scaling of Carbon Nanotube Transistors." Nature Nanotechnology 5 (12): 858-62.

[17] Srivastava, A., Marulanda, J. M. and Xu, Y. et al. 2009. "Current Transport Modeling of Carbon Nanotube Field Effect Transistors." Physica Status Solidi (a) 206 (7): 1569-78.

[18] Zhang, R., Zhang, Y. and Zhang, Q. et al. "Growth of Half-Meter Long Carbon Nanotubes Based on Schulz-Flory Distribution.” Acs Nano 7 (7): 6156-61. 